\title{
Elevated Peripheral Myelin Protein 22, Reduced Mitotic Potential, and Proteasome Impairment in Dermal Fibroblasts from Charcot-Marie-Tooth Disease Type 1A Patients
}

\author{
Sooyeon Lee, ${ }^{*}$ Hannah Bazick, ${ }^{*}$ Vinita Chittoor-Vinod, ${ }^{*}$ Mohammed Omar Al Salihi, ${ }^{*}$ Guangbin Xia, ${ }^{* \dagger}$ and Lucia Notterpek ${ }^{* \dagger}$
}

From the Departments of Neuroscience* and Neurology, ${ }^{\dagger}$ College of Medicine, University of Florida, Gainesville, Florida

Accepted for publication

October 27, 2017.

Address correspondence to Lucia Notterpek, Ph.D., Department of Neuroscience, College of Medicine, University of Florida, 1149 S. Newell Dr., PO Box 100244, Gainesville, FL 32611. E-mail: notterpek@ ufl.edu.

\begin{abstract}
A common form of hereditary autosomal dominant demyelinating neuropathy known as Charcot-MarieTooth disease type 1A (CMT1A) is linked with duplication of the peripheral myelin protein 22 (PMP22) gene. Although studies from animal models have led to better understanding of the pathobiology of these neuropathies, there continues to be a gap in the translation of findings from rodents to humans. Because PMP22 was originally identified in fibroblasts as growth arrest specific gene 3 (gas3) and is expressed broadly in the body, it was tested whether skin cells from neuropathic patients would display the cellular pathology observed in Schwann cells from rodent models. Dermal fibroblasts from two CMT1A pedigrees with confirmed PMP22 gene duplication were studied. Samples from age-matched nonneuropathic individuals were used as controls. CMT1A patient-derived cultures contain approximately 1.5-fold elevated levels of PMP22 mRNA, exhibit reduced mitotic potential, and display intracellular protein aggregates as compared to cells from unaffected individuals. The presence of cytosolic PMP22 coincides with a decrease in proteasome activity and an increase in autophagy-lysosomal proteins, including LC3-II and LAMP1. These results indicate that the abnormalities in the subcellular processing of excess PMP22 elicit a detectable response in human CMT1A fibroblasts, a phenotype that resembles Schwann cells from neuropathic mice. These findings support the use of human CMT1A fibroblasts as a platform for therapy testing. (Am J Pathol 2018, 188: 728-738; https://doi.org/10.1016/ j.ajpath.2017.10.021)
\end{abstract}

Charcot-Marie-Tooth (CMT) neuropathies, with a frequency of approximately 1 in 2500 , comprise a heterogeneous group of disorders, with typical onset in adulthood. Clinical phenotypes of these neuropathies include slowed nerve conduction velocity, muscle weakness and atrophy, and sensory and motor disturbance. ${ }^{1}$ Disease progression and severity are known to vary among individuals and even within families. Among CMT neuropathies, type 1A comprises the largest fraction, a majority of which have been linked with a $1.5-\mathrm{Mb}$ duplication on the short arm of the human chromosome $17 .^{2}$ The peripheral myelin protein 22 (PMP22) gene was mapped to this duplicated region, which results in three copies of the gene. ${ }^{3}$ Although PMP22 is expressed broadly in the body, affected individuals present with a peripheral neuropathy that varies in severity.
PMP22 was first discovered in peripheral nerves as an abundant glycoprotein named PAS- $\mathrm{II}^{4}$ and then cloned from NIH3T3 fibroblasts as growth arrest specific gene 3 (gas-3). ${ }^{5}$ Subsequently, PMP22 was identified as a $22-\mathrm{kDa}$ transmembrane protein constituent of PNS myelin, a cell surface glycoprotein expressed mostly in myelinating Schwann cells. ${ }^{6}$ In peripheral nerves, PMP22 is enriched in the compact portion of Schwann cell myelin, where it is believed to be a structural component. ${ }^{7,8}$ Besides having roles in the process of myelination, the expression level of PMP22 has been shown to impact cell cycle progression, as well as cell

Supported in part by NIH grant NS041012 and the College of Medicine of the University of Florida (L.N.).

Disclosures: None declared. 
spreading and migration. ${ }^{8-10}$ Aberrant expression of PMP22 is also known to impact cellular proteostasis as the overproduced or mutated protein is prone to aggregation. ${ }^{11,12}$ In animal models of CMT1A, affected nerves contain abnormal cytosolic aggregates of PMP22, which are reactive for ubiquitin and are surrounded by autophagic-lysosomal structures. ${ }^{13}$ Cytosolic accumulation of PMP22 in Schwann cells from a CMT1A patient has also been observed, ${ }^{14}$ indicating that elevated expression of PMP22 elicits a detectable subcellular phenotype.

A number of spontaneous and genetically engineered animal models that incorrectly express PMP22 replicate the defects seen in neuropathic patients, including slowed nerve conduction velocity, dysmyelinated and demyelinated axons, overproliferating Schwann cells, and excess deposits of endoneurial tissue. ${ }^{9,15,16}$ The animal models have allowed mechanistic investigations of the neuropathies, as well as therapy testing, yet the findings from the rodent studies have not translated to humans. The disappointing results from the multicenter ascorbic acid trial, ${ }^{17}$ which was based on preclinical studies in mice, ${ }^{18}$ recommend the need for additional models for therapy testing.

Although peripheral nerves are accessible for biopsy, and human Schwann cells survive in culture, ${ }^{19}$ performing nerve biopsies in neuropathic patients might not be ideal due to potential complications. Because dermal fibroblasts from genetic diseases offer an amenable model for investigations of cellular pathology, ${ }^{20}$ cells from genetically diagnosed CMT1A patients were examined. Here, we show that compared to nondiseased human fibroblasts, cells from neuropathic patients are slow to proliferate, express elevated levels of PMP22 mRNA, and contain ubiquitin-reactive proteasome substrates and abnormal cytosolic PMP22 aggregates. These subcellular changes lead to decreased efficiency in proteasome activity and an up-regulation of autophagy-lysosomal proteins.

\section{Materials and Methods}

\section{Fibroblast Cultures}

Human skin fibroblasts from four CMT1A patients (GM05148, GM05167, GM05146, and GM05165) were purchased from the Coriell Institute (Camden, NJ). Skin fibroblasts from non-neuropathic individuals were obtained from either the Coriell Institute (GM11091), or from volunteers under an institutional review board-approved protocol at the University of Florida (Gainesville, FL; MD09, MD13, and MD16) (Table 1). All procedures with the human cells were performed in compliance with institutional review board-approved procedures. Cultures were maintained in Dulbecco's modified Eagle's medium supplemented with $15 \%$ to $20 \%$ fetal bovine serum and 20 $\mathrm{mmol} / \mathrm{L}$ L-glutamine (Gibco, Gaithersburg, MD). Cells were expanded once per week by dissociation with $0.05 \%$ trypsin and used for experiments under 10 passages.
Table 1 List of Studied Cells with Donor Information

\begin{tabular}{lll}
\hline Sample & Age, years & Sample ID \\
\hline Control 1 (C1) & 17 & GM11091 \\
Control 2 (C2) & 21 & MD13 \\
Control 3 (C3) & 34 & MD16 \\
Control 4 (C4) & 56 & MD09 \\
CMT1A Patient 1 (P1) & 17 & GM05148 \\
CMT1A Patient 2 (P2) & 28 & GM05167 \\
CMT1A Patient 3 (P3) & 40 & GM05146 \\
CMT1A Patient 4 (P4) & 51 & GM05165 \\
\hline
\end{tabular}

\section{Cell Proliferation Studies}

Fibroblasts on poly-L-lysine-coated coverslips $\left(10^{5} \mathrm{cells} / \mathrm{cm}^{2}\right)$ were maintained in normal growth medium for 24 hours before incubation with bromodeoxyuridine (BrdU; Roche, Nutley, NJ). After a 4-hour incubation with BrdU, the cells were fixed with acetone/methanol (1:3) for 20 minutes, denatured in $2.5 \mathrm{~N}$ $\mathrm{HCl}$ for 30 minutes at $37^{\circ} \mathrm{C}$, rinsed in phosphate-buffered saline (PBS), and processed for immunolabeling with mouse anti-BrdU (Millipore, Billerica, MA) and rabbit anti-Ki67 (EnCor, Gainesville, FL) antibodies, overnight at $4^{\circ} \mathrm{C}$. Bound primary antibodies were detected with Alexadye-conjugated goat anti-mouse and goat anti-rabbit secondary antibodies (Invitrogen, Carlsbad, CA). Nuclei were stained with Hoechst dye (Invitrogen) and coverslips were mounted with ProLong Antifade mounting medium (Invitrogen). For quantification, BrdU- and Ki67-positive cells in 12 to 70 fixed fields $\left(0.8 \mathrm{~mm}^{2}\right)$ were counted and divided by the total number of cells (Hoechst dye-positive).

\section{Real-Time PCR}

Confluent cultures, after 48 hours incubation in $2 \%$ FCS containing growth medium, were harvested in QIAzol lysis reagent (Qiagen, Germantown, MD), and total RNA was isolated as per the manufacturer's instructions. Total RNA (900 ng) was reverse transcribed using the High-Capacity RNA-to-cDNA kit (Applied Biosystems, Foster City, CA). For quantitative real-time PCR analyses, undiluted cDNA and primers specific for the human PMP22 or glyceraldehyde-3phosphate dehydrogenase (GAPDH; Real Time Primers, Elkins Park, PA) were used with the LightCycler 480 SYBR Green I Master. The following forward: 5'-GTATCATCGTCCTCCACGTC-3', and reverse: 5'-GGCAGAAGAACAGGAACAGA- $3^{\prime}$ primers were used. Primers were designed to amplify the coding region of the human $P M P 22$ gene, recognizing both exon $1 \mathrm{~A}-$ and exon $1 \mathrm{~B}-$ containing transcripts. ${ }^{21}$ The relative expression of PMP22 to GAPDH mRNA was determined using the $2^{-\Delta \Delta C T}$ method. ${ }^{22}$

\section{Immunolabeling with Antibodies and Organelle Markers}

Cells, plated on poly-L-lysine-coated glass coverslips at $10^{5}$ cells $/ \mathrm{cm}^{2}$, were maintained in normal growth media until 
approximately $80 \%$ confluence. For all immunolabeling experiments other than vimentin, the cells were maintained in Dulbecco's modified essential medium supplemented with $2 \%$ fetal bovine serum for the last 48 hours. Subsequently, the samples were fixed with $2 \%$ paraformaldehyde in $0.1 \mathrm{~mol} / \mathrm{L}$ PBS for 20 minutes, followed by rinses in PBS and permeabilization with $0.2 \%$ Triton-X 100 in PBS for 10 minutes, all at $25^{\circ} \mathrm{C}$. After blocking in $5 \%$ normal goat serum diluted in PBS, the cells were incubated with primary antibodies (Table 2) overnight at $4^{\circ} \mathrm{C}$. To detect PMP22, a rabbit polyclonal antibody against a 16-amino acid peptide corresponding to the second extracellular loop of the human PMP22 was used. ${ }^{16,23} \mathrm{~A}$ mouse monoclonal anti-PMP22 antibody (Chemicon, Temecula, CA) was used for double immunostaining with polyclonal LC3 or LAMP1. To label the Golgi, cells were incubated with fluorescein isothiocyanate-conjugated Vicia Villosa Lectin (Invitrogen). ${ }^{24}$ Fluorochrome-conjugated secondary antibodies (goat anti-rabbit and goat antimouse) were purchased from Invitrogen and were diluted in PBS with 5\% normal goat serum. Nuclei were stained with Hoechst dye (Invitrogen). Coverslips were mounted using ProLong Antifade mounting medium (Invitrogen). Images were acquired on a Leica Spinning disk confocal microscope (Leica, Wetzlar, Germany), and formatted in Adobe Photoshop software version 5.5 (Adobe Systems, San Jose, CA).

\section{Biochemical Assays}

For Western blotting, mouse sciatic nerve lysates from wildtype, C22, or PMP22-knockout mice ( $4 \mu \mathrm{g} / \mathrm{lane}$ ) were blotted with anti-human PMP22-specific polyclonal antibody. ${ }^{25}$ Confluent cultures of dermal fibroblasts, after 48 hours incubation in $2 \%$ FCS containing medium, were lyzed in sample buffer $[62.5 \mathrm{mmol} / \mathrm{L}$ Tris, $3 \%$ SDS, and $10 \%$ glycerol, with complete protease (Roche) and phosphatase (Sigma-Aldrich, St. Louis, MO) inhibitors]. Protein concentration was determined using the Bradford method (Pierce Coomassie Plus; Thermo Fisher Scientific, Waltham, MA), and samples were denatured by adding $\beta$-mercaptoethanol and boiling for 10 minutes. Equal protein amounts were resolved on SDS polyacrylamide gels and transferred to polyvinylidene difluoride membranes. Membranes were rinsed in Tris-buffered saline with $0.05 \%$ Tween-20, blocked in 5\% nonfat milk, and then probed with the indicated primary antibodies (Table 2) overnight at $4^{\circ} \mathrm{C}$. Bound antibodies were detected using Western Lightning Plus ECL reagents (Perkin Elmer, Waltham, MA). Films were digitally imaged using a GS-710 densitometer (Bio-Rad Laboratories, Hercules, CA) or a ChemiDoc MP Imaging System (Bio-Rad Laboratories) and analyzed using ImageJ software version 1.45 (NIH, Bethesda, MD; http://imagej.nih.gov/ij). All biochemical experiments were repeated at least three times using protein lysates from independent cultures.

\section{Ub-GFP Transfection}

A fixed number of cells $\left(4 \times 10^{6}\right)$ were transfected with either the green fluorescent protein (GFP)-containing vector $\mathrm{Ub}$ G76V-GFP $^{26}$ or pmaxGFP (Lonza, Basel, Switzerland) plasmids using the Amaxa Lonza Nucleofector kit (VPD1001), as per the manufacturer's instructions. Cells were plated on poly-L-lysine-coated glass coverslips, and the medium was replaced after 3 hours. Electroporation efficiency was determined by counting the pmaxGFP-positive cells, 24 hours post-transfection. To a subset of Ub-G76VGFP samples, we added lactacystin $(10 \mu \mathrm{mol} / \mathrm{L}$;

Table 2 List of Antibodies Used in This Study

\begin{tabular}{|c|c|c|c|c|c|}
\hline \multirow[b]{2}{*}{ Species } & \multirow[b]{2}{*}{ Antigen } & \multirow[b]{2}{*}{ Source } & \multirow[b]{2}{*}{ Catalog no. } & \multicolumn{2}{|l|}{ Dilution } \\
\hline & & & & WB & IF \\
\hline Mouse & BrdU & Millipore clone $131-14,871$ & MAB4072 & NA & $1: 1000$ \\
\hline Mouse & Vimentin & Sigma-Aldrich & V5255 & NA & $1: 1000$ \\
\hline Rabbit & Hu-PMP22 & References 16 and 23 & NA & $1: 1000$ & $1: 1000$ \\
\hline Mouse & $\alpha$-Tubulin & Sigma-Aldrich & T6199 & $1: 5000$ & NA \\
\hline Rabbit & Ubiquitin & EnCor Biotechnology Inc. & RPCA-Ubi-1 & $1: 2000$ & NA \\
\hline Rabbit & GAPDH & EnCor Biotechnology Inc. & RPCA-GAPDH & $1: 30,000$ & NA \\
\hline Rabbit & LAMP1 & Abcam & Ab24170 & $1: 1000$ & NA \\
\hline Rabbit & p62 & Biomol & F48010 & $1: 1000$ & NA \\
\hline Rabbit & TFEB N-Terminal & Sigma-Aldrich & SAB4503154 & $1: 1000$ & NA \\
\hline Rabbit & Cathepsin D & Fitzgerald Industries International & 20C-CR2013RP & NA & $1: 500$ \\
\hline
\end{tabular}

GAPDH, glyceraldehyde-3-phosphate dehydrogenase; IF, immunofluorescence; NA, not applicable; PDI, protein disulfide-isomerase; WB, Western blot. 
Calbiochem, San Diego, CA) as a positive control for proteasome inhibition (data not shown). Hoechst dye was used to label the nuclei, and cell images were acquired 24 hours posttransfection with a SPOT digital camera (Diagnostic Instrumentals, Sterling Heights, MI) attached to a Nikon Eclipse E800 microscope (Nikon, Tokyo, Japan). GFPpositive cells were graphed as the percentage of total (Hoechst-positive) cells.

\section{Statistical Analysis}

For all experiments, means \pm SEM were calculated and significance determined by performing unpaired two-tailed $t$-tests, using GraphPad Prism software version 5 (GraphPad Software, La Jolla, CA). $P<0.05$ was considered significant.

\section{Results}

\section{Altered Mitotic Potential of Fibroblasts from CMT1A Patients}

In characterizing primary skin-derived fibroblasts from CMT1A patients with verified PMP22 gene duplication, the proliferation characteristics of the cultures were examined. Cells from four patients, representing two different families, were assigned patient (P) 1 to 4 in ascending order of donor age (Table 1). Patients 1 (GM05148) and 3 (GM05146) represent a 17-year-old son and his 40-year-old mother, whereas patient 2 (GM05167) and 4 (GM05165), represent a 28-year-old daughter and her 51-year-old father from a second donor family. According to the information provided by Coriell Institute, multiplex ligation-dependent probe amplification was used to confirm the duplication (17p) of the $P M P 22$ gene in all four individuals, and no mutations in the myelin protein zero $(M P Z)$ gene. Clinically, the older patient from each family (Patients 2 and 4 ) were stated to be more affected than their children (Patients 1 and 3) at the time of biopsy. As controls, skin fibroblasts from nonneuropathic individuals with closely matched donor age range of 17 to 56 years were studied (Table 1). Because disease phenotypes in primary skin fibroblasts can disappear with extended culture time, ${ }^{27}$ all data were collected from samples under 10 passages.

To analyze the mitotic state of the studied fibroblasts, the incorporation of BrdU was measured after a 4-hour incubation in serum-rich (15\% to $20 \%$ fetal bovine serum) medium. Analyses of BrdU uptake in control cultures resulted in a range from $30 \%$ to $60 \%$, without a clear agedependency (Figure 1A). Unexpectedly, the youngest donor-derived $\mathrm{C} 1$ cells had a low BrdU incorporation rate, which may be related to this individual having Alagille syndrome, per information from Coriell. Comparisons between patient ( $\mathrm{P} 1$ to $\mathrm{P} 4)$ and control $(\mathrm{C} 2$ to $\mathrm{C} 4)$ samples detected a significant decrease in BrdU incorporation by the CMT1A cells (Figure 1A). In addition, a statistically significant age-associated reduction in BrdU incorporation was found within each of the two affected families. Over $50 \%$ fewer mitotic cells were detected in cultures from patient 3 as compared to patient $1[15.4 \% \pm 2.0 \%$ versus $38.6 \% \pm 2.5 \%$ (means $\pm \mathrm{SEM}$ ); $P=5.38 \mathrm{E}-10$ by $t$-test $]$. Similarly, BrdU uptake decreased 3.7-fold between patients 4 and $2[5.9 \% \pm 0.7$ versus $21.7 \% \pm 2.4$ (means \pm SEM); $P=3.2$ E-06 by $t$-test].

As an additional assay on the proliferation characteristics, the samples were labeled for $\mathrm{Ki}-67$, a nuclear protein expressed in nonquiescent cells (Figure 1B). In agreement with the BrdU-incorporation results (Figure 1A), there were significantly fewer Ki-67-positive mitotic cells in the $\mathrm{C} 1$ and all affected cultures (P2 to $\mathrm{P} 4$ ), relative to age-matched controls. A significant reduction in total mitotic cells was also detected within each family $(P<0.001$ for $\mathrm{P} 1$ versus $\mathrm{P} 3$, and for $\mathrm{P} 2$ versus $\mathrm{P} 4)$. Although the proliferation rates were thus significantly reduced in patient cells, the general cell morphology across control and affected cultures appeared similar (Figure 1C). Immunolabeling with an antivimentin antibody did not show pronounced differences in cell shape or size between control and neuropathic samples (Figure 1C), although heterogeneity in cell size was observed. Together, these data indicate that skin fibroblasts from CMT1A patients display a reduction in mitotic potential.

\section{PMP22 Expression and Localization in Fibroblasts from CMT1A Patients}

To determine whether the CMT1A gene duplication leads to a detectable PMP22 expression phenotype in skin fibroblasts, quantitative real-time PCR was performed. For this assay, the cells were cultured in reduced serum medium ( $2 \%$ fetal bovine serum) for 48 hours before analyses, to promote $P M P 22$ gene expression ${ }^{28}$ and to minimize differences in mitotic phenotypes. PMP22 mRNA measured by real-time PCR indicated approximately 1.5 -fold higher expression in samples from the patients ( $\mathrm{P} 1$ to $\mathrm{P} 4)$ relative to controls $(\mathrm{C} 1$ to $\mathrm{C} 4$ ) (Figure 2A). Having identified low mitotic potential (Figure 1, A and B) and low PMP22 expression in the youngest control cells (C1), this sample was excluded from further analysis.

In parallel with the PMP22 mRNA expression studies, the subcellular localization of PMP22 was examined in the dermal fibroblasts by immunofluorescence, using a polyclonal antibody against a 16-amino acid portion of the human protein. ${ }^{13}$ To document the preferential reactivity of this antibody with the human peptide, nerve lysates from human-PMP22-overexpressing $\mathrm{C} 22{ }^{29}$ wild-type, and PMP22-null mice ${ }^{30}$ were probed (Figure 2B). As shown on the Western blot, a prominent approximately $22-\mathrm{kDa}$ band was identified in nerve samples from the $\mathrm{C} 22$ mice, whereas a fainter band was detected in the wild-type sample. The specificity of the antibody is supported by the lack of reactivity with the PMP22-deficient nerve sample. Using 
A

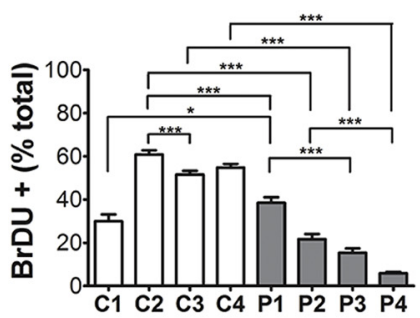

C
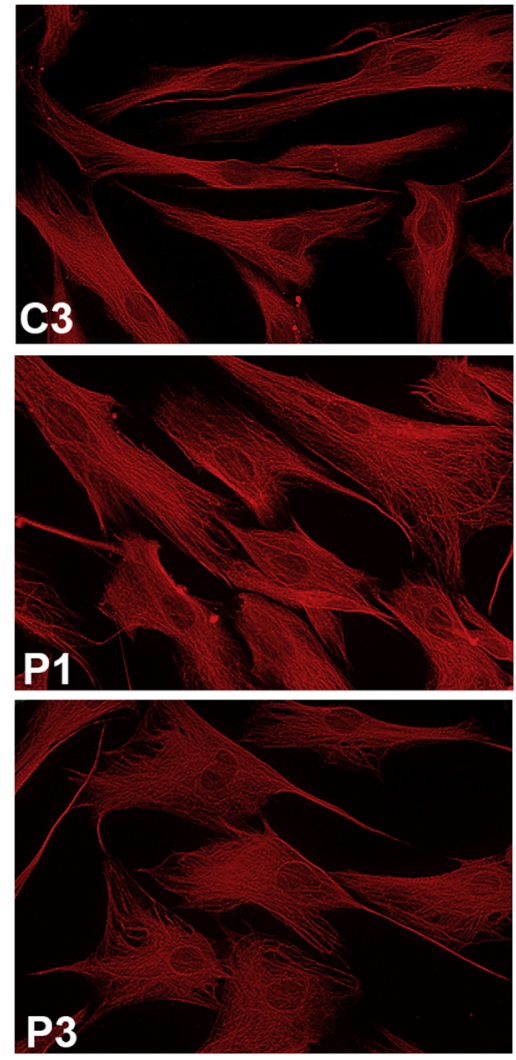

B

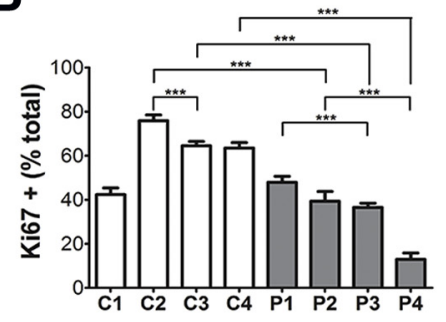

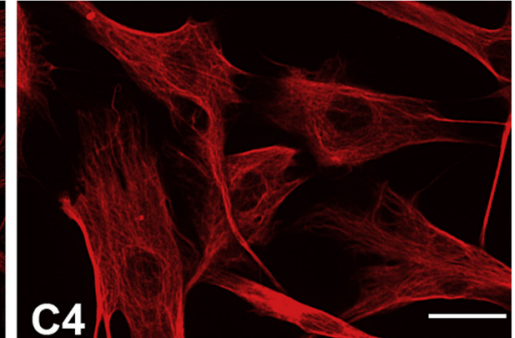
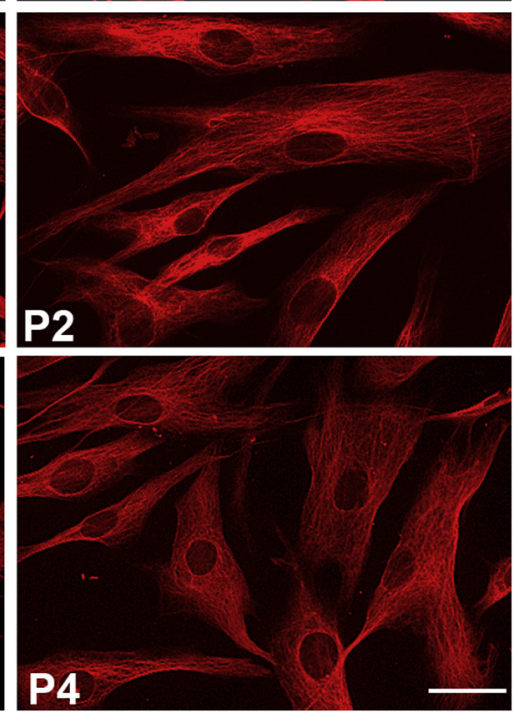

Figure 1 Growth characteristics and cellular morphology of dermal fibroblasts from CMT1A patients. A and $\mathbf{B}$ : Quantification of the percentage of cells with incorporated BrdU (A), or Ki67 (B) reactivity in cultures from control individuals (C1-C4) and CMT1A patients (P1-P4). C: Representative micrographs of antivimentin immunofluorescence of controls ( $\mathrm{C} 3$ and $\mathrm{C} 4$ ) and CMT1A patient (P1 to $\mathrm{P} 4$ ) fibroblasts, shown in red. All cells display elongated, kite-like morphology that is characteristic of dermal fibroblasts. Data are expressed as means \pm SEM. ${ }^{*} P<0.05,{ }^{* *} P<0.001$ (t-test). Scale bars $=20 \mu \mathrm{m}$. this polyclonal antibody, the subcellular distribution of PMP22 was assessed in control and CMT1A patient fibroblasts (Figure 2C). In control cells (C2 to C4), PMP22 was detected on the plasma membrane and in reticular structures, likely reflecting localization in the endoplasmic reticulum (ER) and Golgi (Figure 2C). In fibroblasts from CMT1A patients (P2 to $\mathrm{P} 4)$, prominent PMP22-like immunoreactivity was dispersed in punctate structures and in large perinuclear aggregates (Figure 2C). The large PMP22containing perinuclear aggregates were only seen in a subset of cells $(<10 \%$ of total), and are reminiscent of the aggresomes formed by proteasome inhibition in normal Schwann cells, ${ }^{11}$ or the spontaneous PMP22 aggregates found in samples from $\mathrm{C} 22$ neuropathic mice. ${ }^{13}$

To delineate the subcellular localization of PMP22 in affected fibroblasts, double labeling was performed with anti-PMP22 antibodies and ER or Golgi markers (Figure 3).
Confocal microscopy was used to analyze cells from an agematched control (C4) and the more affected CMT1A family (P2 and P4), because PMP22 aggregates were most prominent in the elder P4 (Figure 2C). As predicted from previous studies, ${ }^{23}$ PMP22-immunoreactive structures in control cells showed reticular distribution and partially costained with the antibody against the ER enzyme, protein disulfide-isomerase (PDI) (Figure 3A) and the Golgi marker Vicia Villosa Lectin (VVL) (Figure 3E). In CMT1A fibroblasts, reticular PMP22-like immunoreactivity was also colocalized in the ER (Figure 3, B-D) and Golgi compartments (Figure 3, F-H). The large perinuclear aggregates partially colabeled with the ER marker, PDI (Figure 3, B and $\mathrm{C}$ ), whereas the more peripherally dispersed, small clusters of PMP22 aggregates did not (Figure 3, B and D). With regards to potential retention in the Golgi, the larger clusters of PMP22 aggregates were typically excluded from 
A

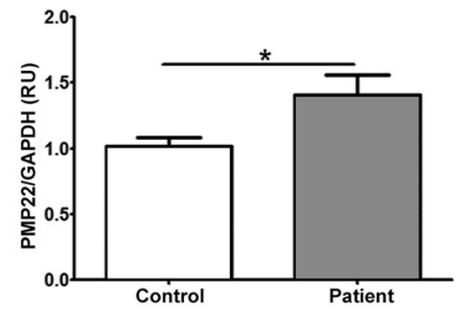

B

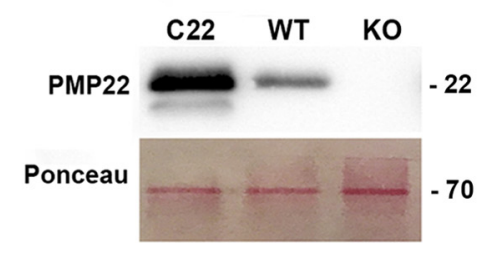

C
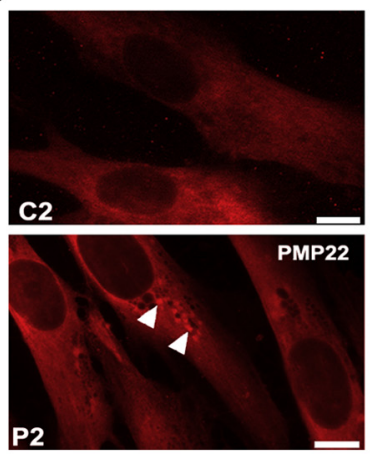
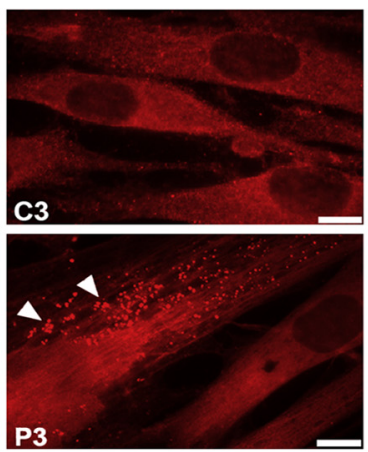
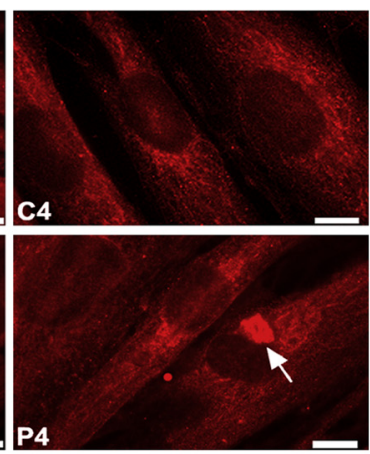

Figure 2 Fibroblasts from CMT1A patients express elevated levels of PMP22 mRNA and exhibit PMP22 protein aggregates. A: PMP22 mRNA levels in control and CMT1A patient fibroblasts, after correction for glyceraldehyde-3-phosphate dehydrogenase (GAPDH), are graphed. Values of the control cells were normalized to 1. B: Sciatic nerve lysates (4 $\mu \mathrm{g} /$ lane) from human-PMP22 overexpressing (C22), wild-type (WT), and PMP22 knockout (KO) mice were immunoblotted with an anti-human PMP22 antibody. Ponceau S staining is shown as control for protein loading. Molecular mass in $\mathrm{kDa}$ is shown on the right. C: Representative micrographs of PMP22-like immunofluorescence in control (C2-C4) and CMT1A patient (P2 $-P 4)$ fibroblasts after labeling with the same antihuman PMP22 antibody, as in B. Intracellular punctate (arrowheads in P2 and P3) and aggregated, perinuclear (arrow in P4) anti-PMP22 antibody reactive structures are marked. $n=3$ independent cultures $(\mathbf{A}) .{ }^{*} P<0.05$ ( $t$-test). Scale bars $=10 \mu \mathrm{m}$. RU, relative units. the Golgi (Figure 3G); however, an occasional PMP22reactive aggregate displayed colabeling with VVL (Figure 3H). The distal, smaller aggregates of PMP22 did not colocalize with the Golgi marker (Figure 3, F and H). These results indicate that a fraction of the overproduced PMP22 is abnormally localized within affected cells, with a likely impact on cellular proteostasis.

\section{Compromised Cellular Proteostasis in CMT1A Fibroblasts}

Because PMP22 is a proteasome substrate, and PMP22 aggregates are reactive for ubiquitin in CMT1A mouse models, ${ }^{11-13}$ double immunolabeling was performed with antiPMP22 and anti-ubiquitin antibodies in patient-derived fibroblasts (Figure 4A). In agreement with results from the animal models, PMP22- and ubiquitin-reactive large perinuclear aggregates (Figure 4A) that were reminiscent of aggresomes formed by pharmacologic inhibition of the proteasome, were observed. ${ }^{11}$ To determine whether the fibroblasts from the patients exhibit deficits in proteasome function, multiple assays were performed (Figure 4, B-G). First, the steady-state levels of polyubiquitinated proteins whose accumulation serves as an index of ubiquitin-proteasome system congestion, was determined. There was an increase in the levels of polyubiquitinated substrates in affected samples, as compared to controls (Figure 4, B and C). Notably, patient cells from low passage numbers contained the highest levels of ubiquitinated aggregates (Figure 4B), with samples from the severely affected P4 patient being most distinguishable. Although variation in polyubiquitinated protein levels with higher cell passages were observed, the blots shown are representative of over 10 independent experiments with cells at passage 6 to 8 (Figure 4C). In agreement with the biochemical studies, immunolabeling with anti-ubiquitin antibody also revealed an abundance of ubiquitin-reactive structures in patient cells, relative to relevant age-matched control (Figure 4D). For quantification of polyubiquitinated proteins, data from independent Western blots were combined and results analyzed for control (C2 to $\mathrm{C} 4)$ and patient (P1 to $\mathrm{P} 4)$ cohorts. There was an approximately 1.5 -fold increase in polyubiquitinated proteins in affected samples, as compared to the control group (Figure 4E).

As an additional assay for proteasome function, cells were transfected with Ubi-GFP reporter, where fluorescence indicates a buildup of nondegraded ubiquitin-tagged substrates. $^{13,25,26}$ Transfection efficiency was monitored with a control GFP-plasmid and was approximately 54\%. Control cells displayed minimal Ubi-GFP fluorescence indicating efficient proteasome function (Figure 4, F and G). In comparison, patient fibroblasts (P2 and P4) displayed a significantly higher percentage of Ubi-GFP-positive cells, which were most pronounced in P4 (Figure 4, F and G). Together, these results indicate that overproduction of PMP22 in primary dermal fibroblasts from CMT1A patients is associated with an impairment of proteasome activity, a cellular phenotype that is consistent between rodent Schwann cells and human fibroblasts.

\section{Autophagy-Lysosomal Response in Neuropathic Samples}

Because previous studies have indicated a prominent role for autophagy in PMP22 aggregate clearance and protein processing, 13,31,32 it was assessed whether the autophagylysosomal pathway is altered in CMT1A cells (Figure 5). 
A

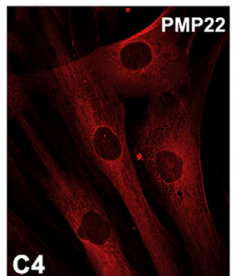

C

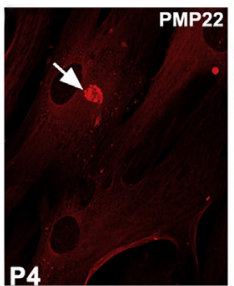

E

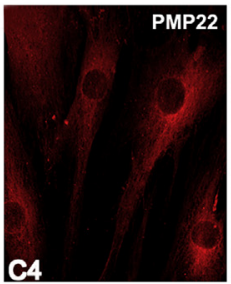

G

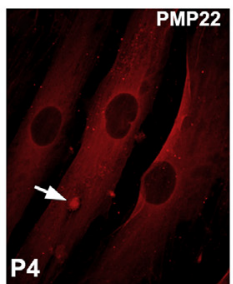

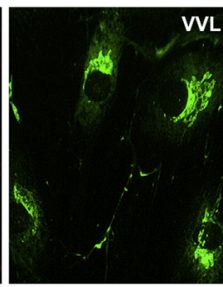
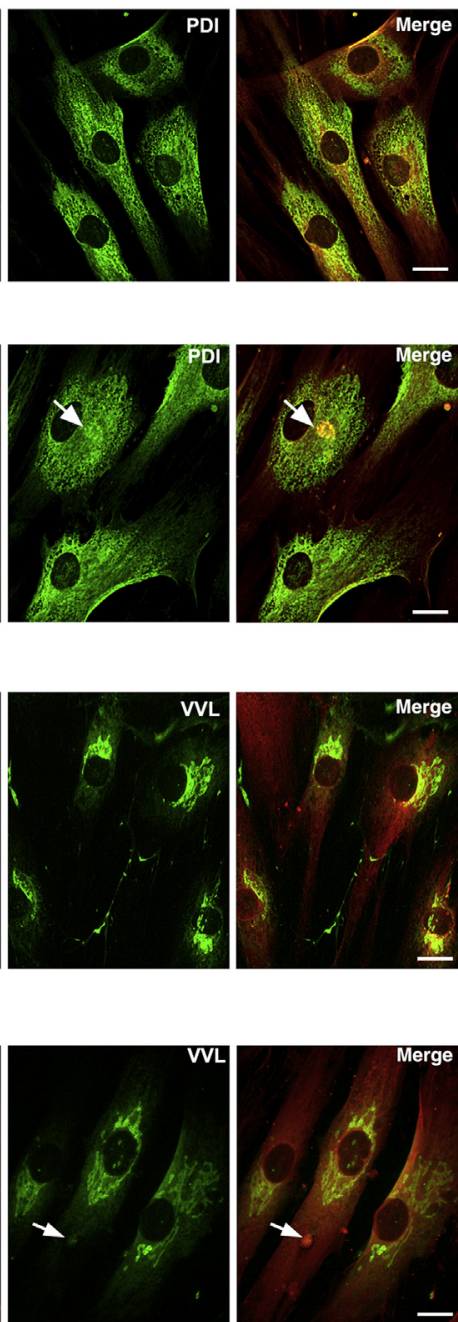

B
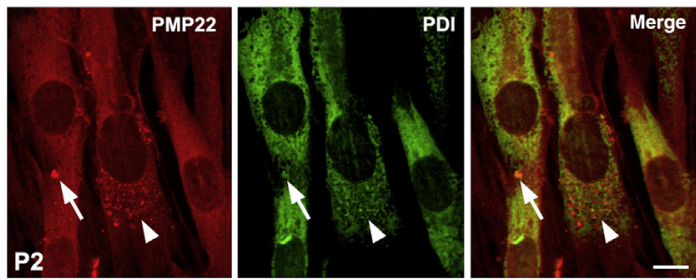

D

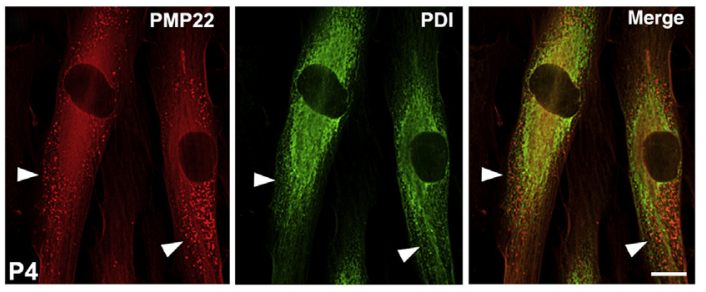

$F$
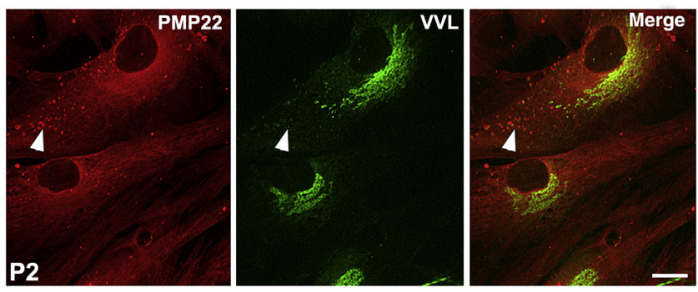

$\mathrm{H}$
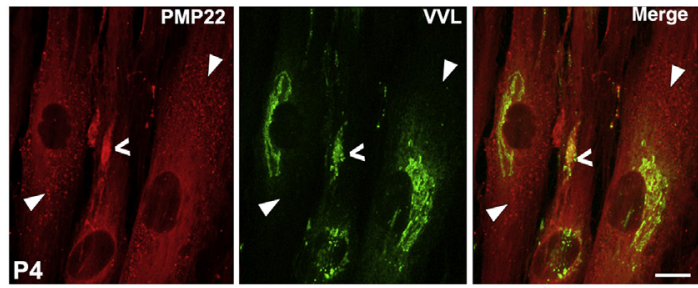

Figure 3 The subcellular localization of the overproduced PMP22. A-D: Anti-PMP22 (red) and anti-protein disulfide isomerase (green) double immunofluorescence shows the distribution of PMP22 relative to the ER in control (C4) (A) and CMT1A patients (P2 and P4) (B-D) fibroblasts. E-H: PMP22 localization (red) and the Golgi marker Vicia Villosa Lectin (green) in control (C4) and CMT1A patients (P2 and P4) fibroblasts are shown. Arrows indicate prominent clusters of PMP22; arrowheads, scattered, punctate anti-PMP22 antibody immunoreactive structures; open arrowheads, the colocalization of PMP22 with the Golgi marker. Scale bars $=20 \mu \mathrm{m}$. PDI, protein disulfide isomerase; VVL, Vicia Villosa Lectin.

The levels and expression of autophagy and lysosomal proteins, including lysosome-associated membrane protein 1 (LAMP1), microtubule-associated protein light chain 3 (LC3), and p62, were determined in samples from control (C2 to $\mathrm{C} 4)$ and neuropathic (P1 to P4) individuals (Figure 5, A-E). The levels of LAMP1 were elevated approximately 1.5-fold in patient samples, compared to the controls (Figure 5, A and B). Within the same cell lysates, the levels of LC3-II, the membrane-associated form of cytosolic LC3-I that is incorporated into the limiting membrane of autophagosomes, was evaluated. ${ }^{33}$ Levels of LC3-II were significantly higher in affected samples relative to controls (Figure 5, A and C). The ubiquitin and LC3 binding protein $\mathrm{p} 62^{34}$ was also analyzed as a measure for autophagy clearance. Although p62 levels were modestly increased in patient fibroblast lysates, this difference did not reach significance (Figure 5, A and D). Similarly, the expression of transcription factor EB (TFEB), the master regulator of lysosomal and autophagy genes, ${ }^{35}$ remained comparable among the groups (Figure 5, A and E).

To assess the subcellular distribution of autophagiclysosomal compartments, cultures were labeled with LysoTracker Red (Invitrogen) (Figure 5F), which allows the detection of acidic compartments in living cells. Although fibroblasts from C4 and P4 displayed an abundance of perinuclear lysosomes, a subset of patient fibroblasts showed intense LysoTracker-accumulated vesicles. To characterize PMP22 distribution in relation to autophagylysosomal compartments, fibroblasts were co-stained with antibodies against PMP22 and the lysosomal hydrolase cathepsin D (Figure 5G) or autophagosome marker LC3 (Figure 5H). There was a partial colocalization of PMP22 with both LC3 and cathepsin D (Figure 5, G and H). Similar recruitment of autophagosomes and lysosomes near PMP22 aggregates have been observed in Schwann cells that 
A
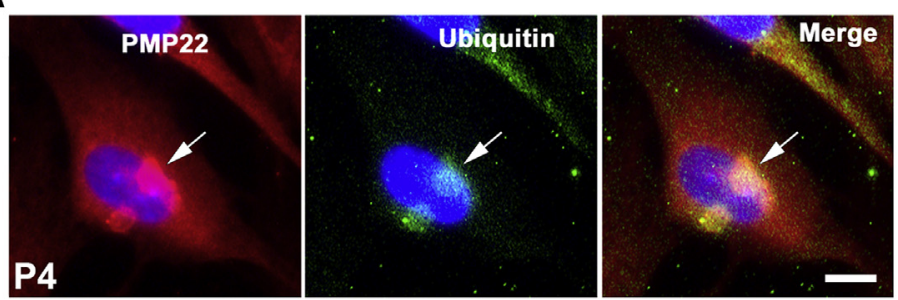

B

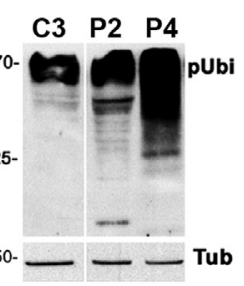

C

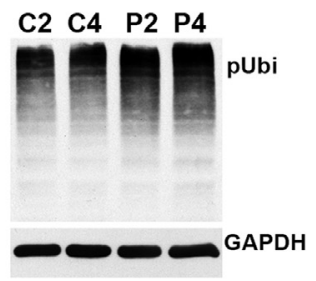

D
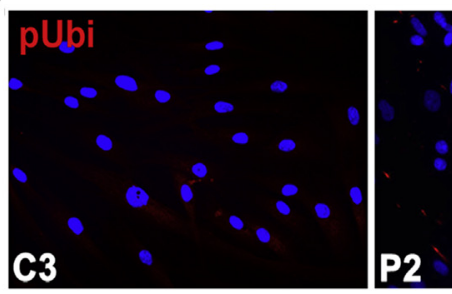

F

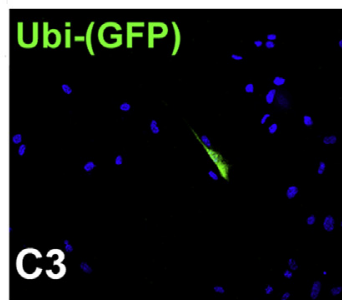

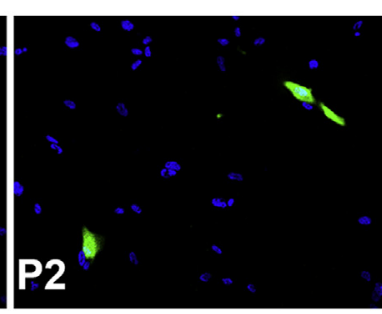

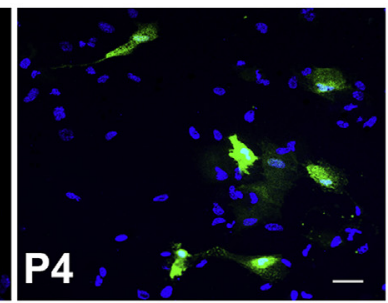

E

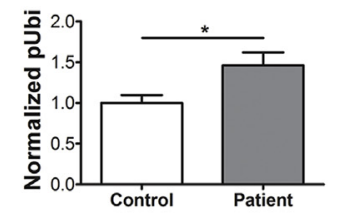

G

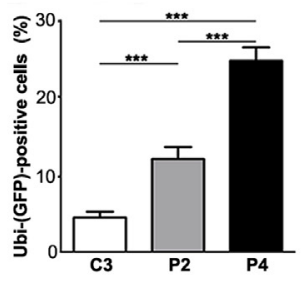

Figure 4 Accumulation of undegraded proteasome substrates in fibroblasts from CMT1A patients. A: Anti-PMP22 and anti-ubiquitin double immunolabeling in P4 fibroblasts. Arrows indicate perinuclear ubiquitin-reactive PMP22 aggregate. B and C: The detection of poly-ubiquitinated substrates in cell lysates from early (B) and late (C) passage control (C2 and C3) and CMT1A (P2 and P4) cultures (20 $\mu \mathrm{g} /$ lane). Tubulin or glyceraldehyde-3-phosphate dehydrogenase (GAPDH) are shown as protein loading controls. D: Immunofluorescence for polyubiquitinated proteins in control (C3) and CMT1A patient fibroblasts (P2 and P4). Inset in P2 indicates no primary antibody control. E: Quantification of ubiquitin immunoblots from control and CMT1A cultures. F: Live-images of cells expressing an Ubi-(GFP) reporter. Nuclei are stained with Hoechst dye (blue). G: Quantification of Ubi-(GFP)-positive cells, represented as a percentage of total. $n=5$ to 6 (E, independent lysates per sample). ${ }^{*} P<0.05,{ }^{* *} P<0.001$ (t-test). Scale bars: $10 \mu \mathrm{m}(\mathbf{A}) ; 50 \mu \mathrm{m}(\mathbf{D}$ and $\mathbf{F})$. pUbi, polyubiquitinated; Tub, tubulin.

expresses mislocalized, excess PMP22. ${ }^{13,36}$ Together, these results indicate that dermal fibroblasts from CMT1A patients share characteristics with murine neuropathic Schwann cells, including the activation and recruitment of autophagy lysosomes to PMP22 aggregates. ${ }^{13,36}$

\section{Discussion}

The results in this study demonstrate that dermal fibroblasts from CMT1A patients display disease phenotypes that are consistent with findings in mouse models of PMP22 neuropathies, including a cellular response to the misexpressed PMP22. ${ }^{12,13}$ In addition, similar to the detection of intracellular PMP22 aggregates seen in these patients' fibroblasts, previous studies in nerve biopsies from CMT1A patients identified abnormally localized, cytosolic PMP22 within Schwann cells. ${ }^{14}$ The fibroblasts also reproduced one of the earliest described effects of PMP22 overexpression, namely, the inverse relationship of high protein levels with reduced cell proliferation. ${ }^{37}$ Nonetheless, dermal fibroblasts lose pathogenic phenotype with passage, which agrees with a report on cells from CMT2L patients. ${ }^{27}$ In this study, the skin fibroblasts retained a pathological phenotype up to 3 months in culture, after which they became indistinguishable from controls. Similar results were observed in the CMT1A fibroblasts, as the ubiquitinated protein accumulation, the prevalence of PMP22 aggregates, as well as the aberrant cell division phenotypes became less noticeable after extended ( 2 to 3 months) culture time.

The understanding of the pathobiology of human disorders, and development of therapies, has been aided by advancements in transgenic animal technologies, yet therapies that appear to work in rodents have not been effective in clinical trials. Studies of disease mechanisms and therapy testing in disease-relevant cell types derived from skin biopsies of affected individuals are of particular interest and now are possible with the induced pluripotent stem cell technology. Because skin fibroblasts can be reprogrammed into Schwann cells, one would expect that they would reproduce a disease-relevant phenotype. Indeed, a recent study by Kitani-Morii et $\mathrm{al}^{38}$ demonstrated that reprogrammed neural crest cells from CMT patients retain diseasespecific molecular features. Nonetheless, successful 
A
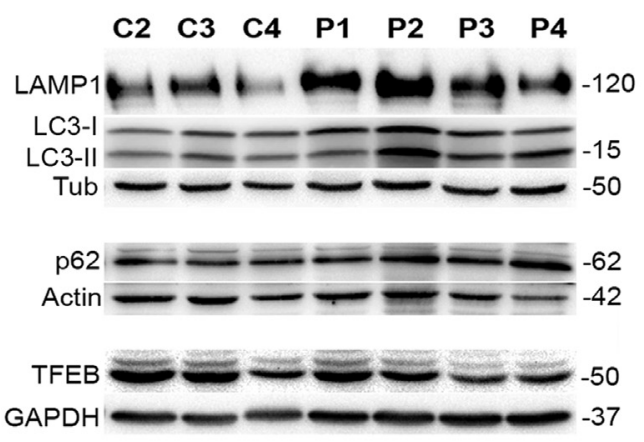

F

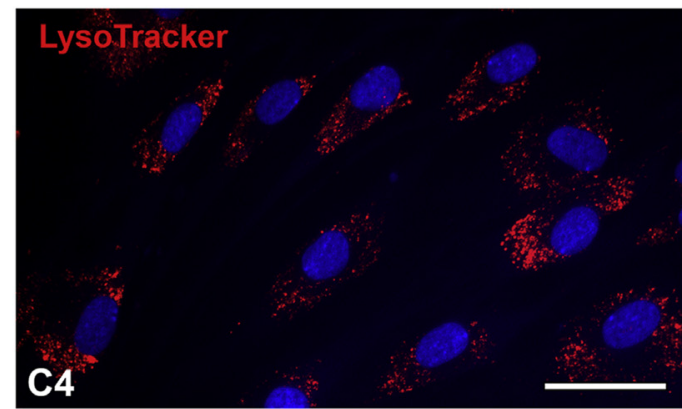

G

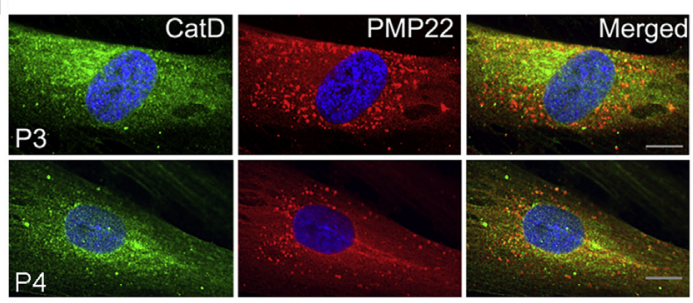

B

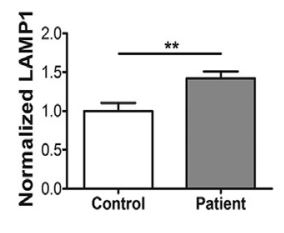

D

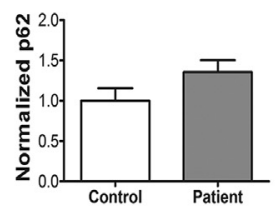

C

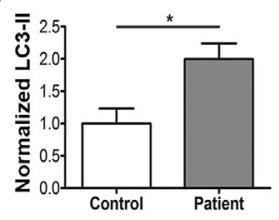

E

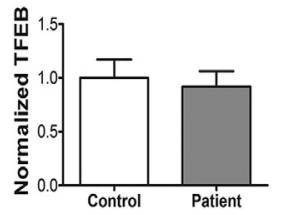

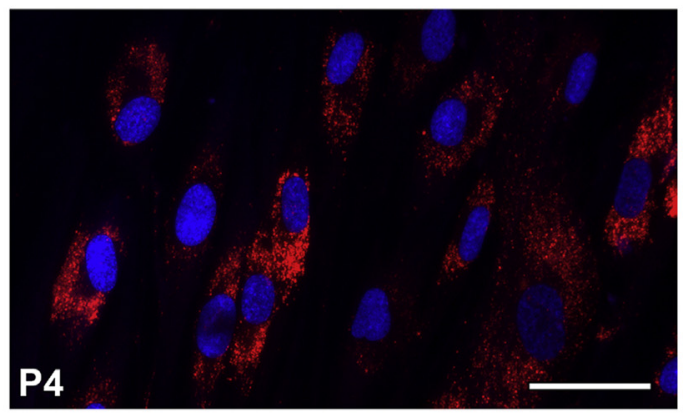

$\mathrm{H}$

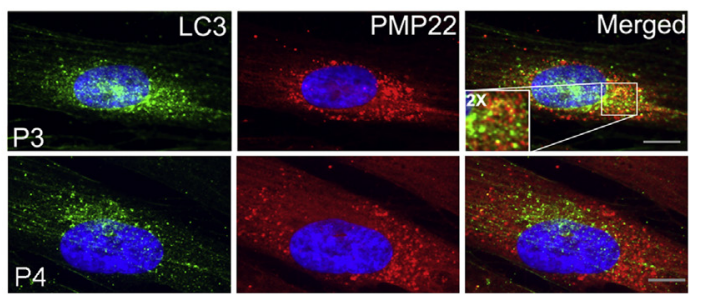

Figure 5 Autophagy-lysosomal response in fibroblasts from CMT1A patients. A: Western blots for LAMP1, LC3-II, p62, and TFEB on whole-cell lysates from the indicated individuals. Each membrane was reprobed with antibodies for a constitutive marker, tubulin, actin, or glyceraldehyde-3-phosphate dehydrogenase (GAPDH). B-E: Quantification of LAMP1, LC3-II, p62, and TFEB protein levels after immunoblotting with the respective antibodies. F: The detection of lysosomes in fibroblasts after labeling with LysoTracker. $\mathbf{G}$ and $\mathbf{H}$ : Confocal images of cells from CMT1A patients (P3 and P4) after double immunolabeling with anti-PMP22 (red) and anti-cathepsin D (G), or anti-LC3 $(\mathbf{H})$ antibodies. In $\mathbf{F}-\mathbf{H}$, nuclei are stained with Hoechst dye (blue). ${ }^{*} P<0.05,{ }^{*} P<0.01(t$-test). Scale bars: $50 \mu \mathrm{m}(\mathbf{F}) ; 10 \mu \mathrm{m}(\mathbf{G}$ and $\mathbf{H})$. CatD, cathepsin D; Tub, tubulin.

reprogramming of CMT1A dermal fibroblasts to functional Schwann cells has not been reported, even though Schwann cells have been derived from human epidermal neural crest and other stem cells. ${ }^{39}$ Schwann cells cultured from CMT1A patient nerve biopsies displayed altered growth behavior, with decreased proliferation in all passages. ${ }^{40}$ These cells also overexpressed PMP22 mRNA, suggesting that glial cells from patients with confirmed PMP22 gene duplication present a disease-relevant phenotype. In comparison to our findings in cultured fibroblasts and the abovecited study in human Schwann cells, ${ }^{40}$ another article reported a lack of correlation between PMP22 transcript levels in skin and nerve biopsies and disease phenotype in a cohort of CMT1A patients. ${ }^{41}$ This later study examined samples from patients in the ascorbic acid trial, and in contrast to the original publication, ${ }^{18}$ they did not report an effect of ascorbate on PMP22 expression. One difference between the mentioned studies and our work is the culturing of the cells in low-serum medium for 48 hours before analysis. This methodology was used because PMP22 transcript levels are known to increase at growth arrest. ${ }^{37}$ In general, however, PMP22 message levels appear to vary in human biopsy material, which led to the suggestion that the extra copy of the PMP22 gene disrupts gene regulatory mechanisms. ${ }^{42}$ Furthermore, there are numerous reports on aberrant PMP22 expression in cancer biopsies, including osteosarcoma and breast cancer, ${ }^{43}$ with the levels of PMP22 expression not necessarily correlating with the mitotic state of the cells. Therefore, more studies with larger sample numbers are necessary before the precise utility of CMT1A patient-derived cells for therapy development and testing can be unequivocally established.

A somewhat unexpected and striking finding in our study is the display of disease-state-relevant phenotypes in the cultured cells. The four studied CMT1A cells represent two distinct, genetically identified CMT1A families with a 
parent and child. Within each of the families, cells from the older, more affected individual displayed a more pronounced disease-relevant pathological phenotype, including reduced mitotic potential and cytosolic PMP22 aggregation (Figures 1 and 2). Furthermore, cells from the most severely affected 51-year-old CMT1A patient (P4) showed the most pronounced effect on proteostasis, with highly significant impairment of proteasome activity (Figure 4). In fact, in a previous study, an over 50\% reduction in the chymotrypsinlike activity of the proteasome, and an over threefold increase in undegraded substrates in P4 cells was reported. ${ }^{25}$ Elevating chaperone expression through pharmacologic induction of the heat shock response, alleviated the proteasome dysfunction, supporting the utility of these cells for in vitro drug screening. ${ }^{25}$ In agreement with our current data, aging indeed is a key risk factor in hereditary neurodegenerative disorders, because the efficacies of protein quality control mechanisms, including the proteasome, are known to decline across the lifespan. ${ }^{44}$

In CMT1A, increasing evidence indicates that the disease mechanism involves failure to process and deliver sufficient PMP22 to myelin, which is underlined by defects in the ubiquitin-proteasome system and cellular proteostasis. ${ }^{45}$ When proteasome function is overburdened by excess misfolded proteins or a pharmacologic block, PMP22 abnormally accumulates in the cytosol in aggregated structures. This reflects the importance of ubiquitin-proteasome system function for PMP22 processing, because a large proportion of newly synthesized PMP22 normally undergoes immediate degradation. ${ }^{23}$ Similarly, enhancing the ubiquitin-proteasome system through dietary restriction or pharmacologic activators improved PMP22 processing in mouse CMT1A Schwann cells and increased their myelination capacity. ${ }^{31,46}$ The pathological phenotype of proteasome impairment discovered in mouse models of CMT1A is preserved in fibroblast from neuropathic patients, which signifies the fundamental nature of how cells respond to the overproduced PMP22. Indeed, cellular stress in response to PMP22 overexpression has been observed in many different cell types and can lead to cell death under certain conditions. ${ }^{47}$

The data described suggest that dermal skin fibroblasts provide a suitable model for therapeutic studies of CMT1A. Our findings are consistent with a recent report in fibroblasts from giant axonal neuropathy patients where abnormal intermediate filament organization was retained in the culture model. ${ }^{20}$ This article led to the detection of altered mitochondrial mobility in affected samples and allowed for correction of the defect by silencing the disease gene. Similarly, dermal fibroblasts from CMT1A patients provide an accessible model where suppression of the overproduced PMP22 can be attained and functional correction of the aberrant mitotic phenotype can be assessed. Using such a simple assay, if consistent across a larger sample set, would provide an ideal platform for drug testing and may lead to new candidate molecules.

\section{References}

1. Saporta MA, Shy ME: Inherited peripheral neuropathies. Neurol Clin 2013, 31:597-619

2. DiVincenzo C, Elzinga CD, Medeiros AC, Karbassi I, Jones JR, Evans MC, Braastad CD, Bishop CM, Jaremko M, Wang Z, Liaquat K, Hoffman CA, York MD, Batish SD, Lupski JR, Higgins JJ: The allelic spectrum of Charcot-Marie-Tooth disease in over 17,000 individuals with neuropathy. Mol Genet Genomic Med 2014, 2:522-529

3. Patel PI, Roa BB, Welcher AA, Schoener-Scott R, Trask BJ, Pentao L, Snipes GJ, Garcia CA, Francke U, Shooter EM, Lupski JR, Suter U: The gene for the peripheral myelin protein PMP-22 is a candidate for Charcot-Marie-Tooth disease type 1A. Nat Genet 1992, 1:159-165

4. Kitamura K, Suzuki M, Uyemura K: Purification and partial characterization of two glycoproteins in bovine peripheral nerve myelin membrane. Biochim Biophys Acta 1976, 455:806-816

5. Schneider GB, Gilmore AP, Lohse DL, Romer LH, Burridge K: Microinjection of protein tyrosine phosphatases into fibroblasts disrupts focal adhesions and stress fibers. Cell Adhes Commun 1998, 5: 207-219

6. Snipes GJ, Suter U, Welcher AA, Shooter EM: Characterization of a novel peripheral nervous system myelin protein (PMP-22/SR13). J Cell Biol 1992, 117:225-238

7. Naef R, Suter U: Many facets of the peripheral myelin protein PMP22 in myelination and disease. Microsc Res Tech 1998, 41:359-371

8. Lee S, Amici S, Tavori H, Zeng WM, Freeland S, Fazio S, Notterpek L: PMP22 is critical for actin-mediated cellular functions and for establishing lipid rafts. J Neurosci 2014, 34:16140-16152

9. Jetten AM, Suter U: The peripheral myelin protein 22 and epithelial membrane protein family. Prog Nucleic Acid Res Mol Biol 2000, 64: 97-129

10. Zoidl G, Blass-Kampmann S, D'Urso D, Schmalenbach C, Muller HW: Retroviral-mediated gene transfer of the peripheral myelin protein PMP22 in Schwann cells: modulation of cell growth. EMBO J 1995, 14:1122-1128

11. Notterpek L, Ryan MC, Tobler AR, Shooter EM: PMP22 accumulation in aggresomes: implications for CMT1A pathology. Neurobiol Dis 1999, 6:450-460

12. Fortun J, Dunn WA Jr, Joy S, Li J, Notterpek L: Emerging role for autophagy in the removal of aggresomes in Schwann cells. J Neurosci 2003, 23:10672-10680

13. Fortun J, Go JC, Li J, Amici SA, Dunn WA Jr, Notterpek L: Alterations in degradative pathways and protein aggregation in a neuropathy model based on PMP22 overexpression. Neurobiol Dis 2006, 22: 153-164

14. Nishimura T, Yoshikawa H, Fujimura H, Sakoda S, Yanagihara T: Accumulation of peripheral myelin protein 22 in onion bulbs and Schwann cells of biopsied nerves from patients with Charcot-MarieTooth disease type 1A. Acta Neuropathol 1996, 92:454-460

15. Notterpek L, Tolwani RJ: Experimental models of peripheral neuropathies. Lab Anim Sci 1999, 49:588-599

16. Chittoor VG, Sooyeon L, Rangaraju S, Nicks JR, Schmidt JT, Madorsky I, Narvaez DC, Notterpek L: Biochemical characterization of protein quality control mechanisms during disease progression in the C22 mouse model of CMT1A. ASN Neuro 2013, 5:e0128

17. Lewis RA, McDermott MP, Herrmann DN, Hoke A, Clawson LL, Siskind C, Feely SM, Miller LJ, Barohn RJ, Smith P, Luebbe E, Wu X, Shy ME; Muscle Study Group: High-dosage ascorbic acid treatment in Charcot-Marie-Tooth disease type 1A: results of a randomized, doublemasked, controlled trial. JAMA Neurol 2013, 70:981-987

18. Passage E, Norreel JC, Noack-Fraissignes P, Sanguedolce V, Pizant J, Thirion X, Robaglia-Schlupp A, Pellissier JF, Fontes M: Ascorbic acid treatment corrects the phenotype of a mouse model of Charcot-MarieTooth disease. Nat Med 2004, 10:396-401

19. Levi AD, Burks SS, Anderson KD, Dididze M, Khan A, Dietrich WD: The use of autologous Schwann cells to supplement sciatic nerve repair 
with a large gap: first in human experience. Cell Transplant 2016, 25: $1395-1403$

20. Lowery J, Jain N, Kuczmarski ER, Mahammad S, Goldman A, Gelfand VI, Opal P, Goldman RD: Abnormal intermediate filament organization alters mitochondrial motility in giant axonal neuropathy fibroblasts. Mol Biol Cell 2016, 27:608-616

21. Rao RG, Sudhakar D, Hogue CP, Amici S, Gordon LK, Braun J, Notterpek L, Goodglick L, Wadehra M: Peripheral myelin protein-22 (PMP22) modulates alpha 6 integrin expression in the human endometrium. Reprod Biol Endocrinol 2011, 9:56

22. Livak KJ, Schmittgen TD: Analysis of relative gene expression data using real-time quantitative PCR and the 2(-Delta Delta C(T)) method. Methods 2001, 25:402-408

23. Pareek S, Notterpek L, Snipes GJ, Naef R, Sossin W, Laliberté J, Iacampo S, Suter U, Shooter EM, Murphy RA: Neurons promote the translocation of peripheral myelin protein 22 into myelin. J Neurosci 1997, 17:7754-7762

24. Ryan MC, Notterpek L, Tobler AR, Liu N, Shooter EM: Role of the peripheral myelin protein $22 \mathrm{~N}$-linked glycan in oligomer stability. J Neurochem 2000, 75:1465-1474

25. Chittoor-Vinod VG, Lee S, Judge SM, Notterpek L: Inducible HSP70 is critical in preventing the aggregation and enhancing the processing of PMP22. ASN Neuro 2015, 7. 1759091415569909

26. Dantuma NP, Lindsten K, Glas R, Jellne M, Masucci MG: Short-lived green fluorescent proteins for quantifying ubiquitin/proteasomedependent proteolysis in living cells. Nat Biotechnol 2000, 18: $538-543$

27. Irobi J, Holmgren A, De Winter V, Asselbergh B, Gettemans J, Adriaensen D, Ceuterick-de Groote C, Van Coster R, De Jonghe P, Timmerman V: Mutant HSPB8 causes protein aggregates and a reduced mitochondrial membrane potential in dermal fibroblasts from distal hereditary motor neuropathy patients. Neuromuscul Disord 2012, 22:699-711

28. Fabbretti E, Edomi P, Brancolini C, Schneider C: Apoptotic phenotype induced by overexpression of wild-type gas3/PMP22: its relation to the demyelinating peripheral neuropathy CMT1A. Genes Dev 1995, 9: $1846-1856$

29. Huxley C, Passage E, Manson A, Putzu G, Figarella-Branger D, Pellissier JF, Fontés M: Construction of a mouse model of CharcotMarie-Tooth disease type 1A by pronuclear injection of human YAC DNA. Hum Mol Genet 1996, 5:563-569

30. Amici SA, Dunn WA Jr, Murphy AJ, Adams NC, Gale NW, Valenzuela DM, Yancopoulos GD, Notterpek L: Peripheral myelin protein 22 is in complex with alpha6beta4 integrin, and its absence alters the Schwann cell basal lamina. J Neurosci 2006, 26:1179-1189

31. Rangaraju S, Verrier JD, Madorsky I, Nicks J, Dunn WA Jr, Notterpek L: Rapamycin activates autophagy and improves myelination in explant cultures from neuropathic mice. J Neurosci 2010, 30: $11388-11397$

32. Fortun J, Verrier JD, Go JC, Madorsky I, Dunn WA, Notterpek L: The formation of peripheral myelin protein 22 aggregates is hindered by the enhancement of autophagy and expression of cytoplasmic chaperones. Neurobiol Dis 2007, 25:252-265

33. Mizushima N, Yoshimori T: How to interpret LC3 immunoblotting. Autophagy 2007, 3:542-545

34. Pankiv S, Clausen TH, Lamark T, Brech A, Bruun JA, Outzen H, Øvervatn A, Bjørkøy G, Johansen T: p62/SQSTM1 binds directly to Atg8/LC3 to facilitate degradation of ubiquitinated protein aggregates by autophagy. J Biol Chem 2007, 282:24131-24145

35. Settembre C, Di Malta C, Polito VA, Garcia Arencibia M, Vetrini F, Erdin S, Erdin SU, Huynh T, Medina D, Colella P, Sardiello M, Rubinsztein DC, Ballabio A: TFEB links autophagy to lysosomal biogenesis. Science 2011, 332:1429-1433

36. Notterpek L, Shooter EM, Snipes GJ: Upregulation of the endosomallysosomal pathway in the trembler-J neuropathy. J Neurosci 1997, 17: $4190-4200$

37. Manfioletti G, Ruaro ME, Del Sal G, Philipson L, Schneider C: A growth arrest-specific (gas) gene codes for a membrane protein. Mol Cell Biol 1990, 10:2924-2930

38. Kitani-Morii F, Imamura $\mathrm{K}$, Kondo $\mathrm{T}$, Ohara $\mathrm{R}$, Enami $\mathrm{T}$, Shibukawa R, Yamamoto T, Sekiguchi K, Toguchida J, Mizuno T, Nakagawa M, Inoue H: Analysis of neural crest cells from CharcotMarie-Tooth disease patients demonstrates disease-relevant molecular signature. Neuroreport 2017, 28:814-821

39. Sakaue M, Sieber-Blum M: Human epidermal neural crest stem cells as a source of Schwann cells. Development 2015, 142:3188-3197

40. Hanemann CO, Rosenbaum C, Kupfer S, Wosch S, Stoegbauer F, Muller HW: Improved culture methods to expand Schwann cells with altered growth behaviour from CMT1A patients. Glia 1998, 23:89-98

41. Nobbio L, Visigalli D, Radice D, Fiorina E, Solari A, Lauria G, Reilly MM, Santoro L, Schenone A, Pareyson D; CMT-TRIAAL Group: PMP22 messenger RNA levels in skin biopsies: testing the effectiveness of a Charcot-Marie-Tooth 1A biomarker. Brain 2014, 137:1614-1620

42. Katona I, Wu X, Feely SM, Sottile S, Siskind CE, Miller LJ, Shy ME, Li J: PMP22 expression in dermal nerve myelin from patients with CMT1A. Brain 2009, 132:1734-1740

43. Ashki N, Gordon L, Wadehra M: Review of the GAS3 family of proteins and their relevance to cancer. Crit Rev Oncog 2015, 20:435-447

44. Lee S, Notterpek L: Dietary restriction supports peripheral nerve health by enhancing endogenous protein quality control mechanisms. Exp Gerontol 2013, 48:1085-1090

45. Rangaraju S, Notterpek L: Autophagy aids membrane expansion by neuropathic Schwann cells. Autophagy 2011, 7:238-239

46. Madorsky I, Opalach K, Waber A, Verrier JD, Solmo C, Foster T, Dunn WA Jr, Notterpek L: Intermittent fasting alleviates the neuropathic phenotype in a mouse model of Charcot-Marie-Tooth disease. Neurobiol Dis 2009, 34:146-154

47. Brancolini C, Edomi P, Marzinotto S, Schneider C: Exposure at the cell surface is required for gas3/PMP22 to regulate both cell death and cell spreading: implication for the Charcot-Marie-Tooth type 1A and Dejerine-Sottas diseases. Mol Biol Cell 2000, 11:2901-2914 